\title{
(21) QUANTITATIVE RETROSPECTIVE ANALYSIS OF WATER AND WASTEWATER MANAGEMENT SYSTEMS: \\ A CONCEPTUAL EXPLORATION
}

Masahisa Nakamura*

\begin{abstract}
Plans for water and wastewater management systems are prepared with a long development time in mind. Over the period, a plan undergoes incremental alterations in its substance. These alterations are made, in general, with great emphasis on satisfying the prospective requirements in the coming years, but with little regard to the causes of unfulfillment of or of deviation from the original aspiration. This lack of retrospective analysis may lead to cumulative disorientation in the plan. This paper makes a modest attempt to shed some light on retrospective analysis issues and methodologies.
\end{abstract}

KEYWORDS; water and wastewater management, planning analysis, retrospective analysis, multiobjective analysis

\section{Introduction}

Setting a framework of discussion around the subject of retrospective analysis in planning is an extremely challenging task. Rarely do environmental systems literature dichotomize planning analysis into, for example, prospective analysis versus retrospective analysis. Among the reasons are:

(a) that, by definition, planning is prospective, i.e., you cannot plan the past,

(b) that, planning implies extraporation of the past, i.e., retrospective analysis is an implied and integral part of planning; and

(c) that, because of the above, dealing with the taxonomy of retrospective analysis would be just as overwhelmingly encompassing a task as dealing with the taxonomy of planning analysis.

Thus, the preparation of a sharply focused discussion on aspects of retrospective analysis would be an insurmountable task.

Retrospective analyses are performed under various names and by applying various analysis methodologies. They are performed for the following purposes.

(1) mid-course correction of ongoing plans

In the real world, the plans are plans, and they do not necessarily get realized as planned. The mid-course

* Lake Biwa Research Institute, Shiga Prefecture 
correction, resorting to a mid-term review or a retrospective analysis, is therefore a necessary part of planning. In the private sector planning situations, it is a matter of assessing the degree of deviation from the pre-established norm, and the most important issue here is for the decision-maker to identify the reasons for the deviations so as for him to correct its course. The same is true for the public-sector planning situations, except that there is a greater degree of institutional involvement in the final decision-making, thus rendering lesser degree of freedom in mid-course correction. In addition, the government agencies tends to shay away from having to conduct retrospective analysis, for good reasons. Such analyses may defy the promotion of the plans and projects they are in charge of.

(2) transfer of planning experiences

On a scholarly basis, retrospective analyses are conducted in a variety of ways in a variety of social science fields, basically for extracting generality in scientific observations, for possible transfer of planning experiences form one situation to another, at different times and in different places. Most social science fields are founded on objective assessment of the past, including assessment of plan and projects in retrospect. The transferability of planning experiences depends largely on the the weight of planning factors assessed differently under different circumstances. The methodological development, therefore, vary among different disciplines. Behavioral sciences focus more on the patterns of human behavior under a variety of circumstances, while management sciences focus more on the principles of institutional orientation and motivation.

With these observations in mind, this paper attempts to probe; (a) the operational definition of retrospective analysis, (b) the methods of retrospective analysis in general, (c) the concept of prescriptive retrospective analysis, all with respect to issues related to water and wastewater management.

\section{Operational Definition of Retrospective Analysis}

2. 1 Descriptive Retrospective Analysis

A particular plan gets formulated and implemented along the continuum of time. To analyze it to reflect on the current state of the plan, the situation surrounding the particular time-frame of analysis will have to be properly described. It has to be described with respect to; (a) the physical setup, (b) the institutional setup, (c) the environment, (d) the interacting elements, (e) the overriding driving factors of the plan, etc. The depth and the clarity of description will very much dictate the value of analysis in retrospect. Methodologies have been proposed, developed, refined and used in the fields of science, with different emphasis in different scientific discipline. Structured framework of analysis have 
been proposed, to accommodate for specific types of problems including issues related to management of water and wastewater systems. The analytical framework developed with no particular intention of it being applied for retrospective analysis may turned out to serve well for the purpose.

2. 2 Prescriptive Retrospective Analysis

Planning problems involving location or construction of physical facilities often require prescriptive analysis at the time of the its inception. Planning of water and wastewater management systems often falls into this category of problems. A choice among alternative possibilities with potential conflicts of interest may have to be decided, for example, on the basis of best compromise plan. Or, a choice among alternative possibilities with uncertain future demand conditions may have to be fulfilled, for example, on the basis of robustness analysis of the proposed plan. The methods of analysis best suited to this type of problems belong to what is classified as systems analysis methods.

The prescriptive retrospective analysis, then, will have to be place against these types of prescriptive planning analysis performed at the outset, based on systems analysis methods. In other words, the analysis will have to address "the unfulfilled wishes in retrospect". That is, for example, there are cases in which an alternative course of action;

(a) could have been taken, but was not taken because...

(b) should have been taken, despite...

(c) would have been possible, provided...

etc., implying that in the prescriptive retrospective analysis, one would extrapolate the meaning of the relationship between the past decision and other alternative possibilities more directly than in the case of the descriptive retrospective analysis.

\section{Review of Descriptive Retrospective Analysis Methods}

Associated with most any plan involving water and wastewater management are a large number of quantitative and qualitative planning factors. They are of political, institutional and technological nature, most of them intertwined with eachother. In analyzing the plan in retrospect, therefore, only a subset of factors best suited for the subject discussion is selected to describe a plan and its attributes. Typically, the descriptive retrospective analysis, performed with the aim of clarifying the underlying issues associated with the plan, its strengths and weaknesses, its deviation from a theoretical norm, etc., get performed resorting to some structured analysis procedure.

3. 1 Structured Qualitative Analysis Methods

Presented below are three different structured methods of analysis likely to be usefully applied to retrospective analysis situations. 
A. Assumption Analysis

A structured analysis procedure, called Assumption Analysis, has been developed by Chandler(1) for use in establishing the links among sets of assumptions used by different groups of role-players involved in the analysis of a development plan. He applied the method for analyzing a hydroelectric project in Nepal. Assumptions have been made, for example, through Western engineering experience, in the technical-aid project development process, by foreign consultants, as well as by the host country nationals. Identification of the assumptions and construction of assumption analysis chart, two basic steps among the seven-step procedure, constitute a very well conceived descriptive retrospective analysis of a plan with general applicability with specific intention of transferring experiences from one socio-cultural setup to another.

B. A transaction-based approach to policy intervention

Bryson and Ring(2) presented, in the treatment of relationship among dimensions of transactions, governance structures, and governing principles in public highschool education, application of the transaction-based approach, as theorized by Williamson(3) in his famous treatise of Institutional Economics called the transaction cost economics approach. The proposed method which examines the relative capacity of policy mechanism(bounded rationality, opportunism, uncertainty, assets specificity, frequency, information impactedness, small numbers, excludability, jointness) and governing principles (efficiency, justice, liberty) to resolve transaction dimensions, such as self-service, voluntary arrangement, regulations, markets, vouchers subsidies, government service. The method proposed falls well within the definition of descriptive retrospective analysis mentioned earlier, particularly with respect to institutional issues in the management system. Application of this approach to wateruse conflicts in Asian Metropolices is currently under consideration(4). Analysis of transaction cost, though not as rigorous in the framework of analysis has been presented by Saliba(5) with respect to water markets in the Southwestern States in U.S. A.

C. A multiple Perspective Analysis

Linstone (6) proposes application of a multiple perspective analysis for analyzing retrospectively a plan and its implementation involving man(e.g., vested interest groups), institutions, and the environment. His three perspectives are called technical (T), organizational (O), and personal(P) perspectives. The $T$ perspective sees the world through quantitative analysis (e.g., the curves, computer models, etc.), the $O$ perspective sees the world through an organizational filter (e.g., unique manufacturing division or government agency view, etc.), and the $P$ perspective is the world as seen through the filter of the individual (e.g., intuition, charisma, leadership and self-interest, etc.) His proposal is that $T, O$, 
and $P$ perspectives differ in their scope, orientation, direction and value system, etc., and that clarification of the multiple perspectives greatly help identify issues of significance in prospective planning as well as help analyze past planning situations in retrospect. The dimensions he proposed to be different among $T, O$, and $P$ include, world view, ethical basis, goal, modes of inquiry, time concept, planning horizon, discount rate, constraint setting, behavioral characteristics, and mode of communication. His application example include one on risk analysis.

3. 2 Retrospective Projection
In addition, there are frequent use of retrospective projection for descriptive purposes. Shown below are three typical examples.

A. Use of Time Dependent Physical Model

A physical model verified over time is often used for descriptive retrospective analysis, in which the values of projection are verified against the known values obtained in actual physical measurements. The retrospective implication here is that the model so verified possesses certain capability to extraporate the subject of analysis back to what could have been at a point in the past. (Fig. 1)

B. Use of Bivariate TimeDependent Plot

Two time-dependent variables in combination will create an ideal projection space for retrospective analysis. The deviation from the norm shown in the projection space will imply the condition under which the activity at the time was undertaken. Retrospectively as well as prospectively, the projection is an extraporation of the known relationship between the two exclusive variables. (Fig. 2)

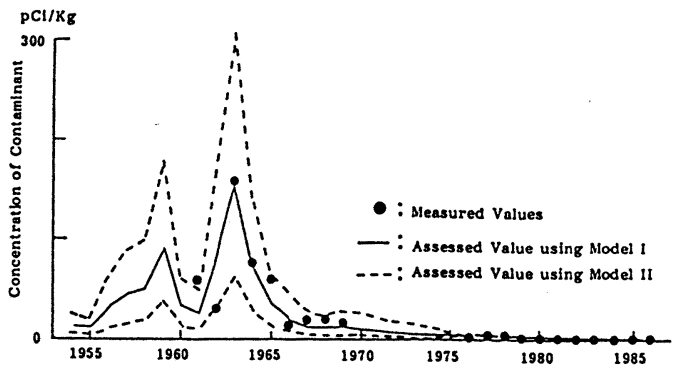

Fig.1 A Time-Dependent Physical Model

Source: Morisawa, S. and Inoue, Y.. Kankyochu deno Busshituido Kalsekiheno Fuzzy Kaisekilyouhou no Katuyou Shuhou nituite, (In Japanese)

Paddy Fleld Area(ha)

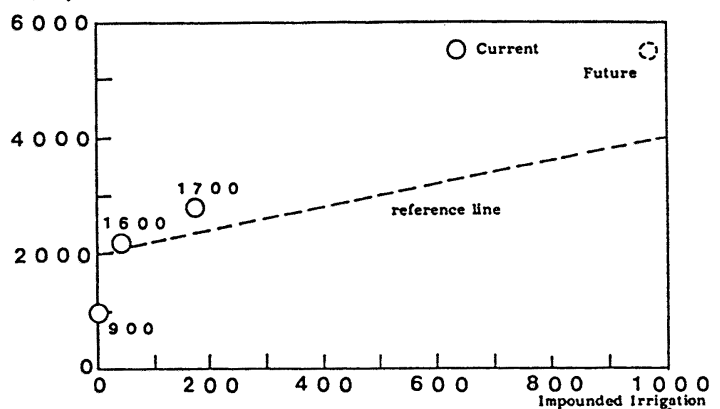

Fig. 2 Bivariate Time-Dependent Plot Water $\left(10,000 \mathrm{~m}^{3}\right)$

Source: Futawatari, T. and I. Kusuda, Change of Water Management System in the Basin of the River Rokkaku, Environment

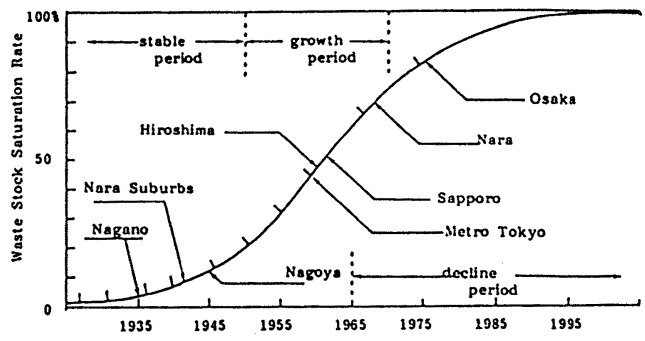

Fig. 3 A. Time-Dependent Socio-economic Indicator Model

Source: T. Sueishl, Toshi Kankyo no Sosel, Chuko Shinsho (1975) (in Japanese) 
C. Use of Time-Dependent Socioeconomic Indicator Model

A socioeconomic attribute as expressed through an index value may be assessed against time to show a hidden trend not apparent in the observation of a single variable. The retrospective implication here is that the well conceived model would have a capability to describe the attribute over a widely different situation such as geographical locations. (Fig. 3)

4. Conceptual Exploration of Prescriptive Retrospective Analysis Systems analysis methods are, by nature, prospective analysis methods. Most of the systems analysis methods are also prescriptive rather than descriptive. Simulation is categorized as descriptive in that it is applied to numerically reproduce the real world phenomena. Most other methods are designed to identify a good, if not optimal, solution of a quantitatively defined system model of the real world planning problem. They generally fall in the category of models called "multiobjective planning models". Our discussion would be pretty much confined to this subset of models.

In bringing in multiobjective systems analysis methods into discussion here, we need to take particular note of the following observations:

(1) there have been continuing soul-searching by the publicsector systems analysis researchers as to whether multiobjective systems analysis planning models have been viable in the process of real-world planning situations, particularly when systems dealt with involve complex social, political and institutional factors.

(2) there is hardly any literature which identifies multiobjective systems analysis models as having the potential of being used for retrospective analysis.

These two observations are in fact closely related.

Inherent in modeling exercises is. a process of abstraction of the complex structure and nature of real-world planning problems. Mathematical description of the real world tend to be oversimplified. For example,

(a) the variables included in the model would only be only approximate representation of the real world variables,

(b) the mathematical description (functional description of objectives and constraints) may be inadequate and often inappropriate, or

(c) computational capability (e.g., the number of objectives which can be included in the analysis) may be far too limited.

The expected end result of the above limitations would be the incorrect projection of the real world objectives on to the objective space of the multiobjective analysis. It would be natural that the revealed reality not likely be the Pareto optimal.

The retrospective analysis of a planning problem based on multiobjective formulation, seems to have a potential of shedding some light on the above. Take the case represented in Fig. 4, for example, in which a set of pseudo Pareto optimal 
solution (approximate noninferior solution set) is represented by square. Suppose this problem was a prospective one (analysis for a particular time in future based on the present data), and suppose the three objectives identified here as $E$, $F t$ and $F c$ as being quite dictating, then the inclination is to choose a plan among the solution set. But if this was the result of

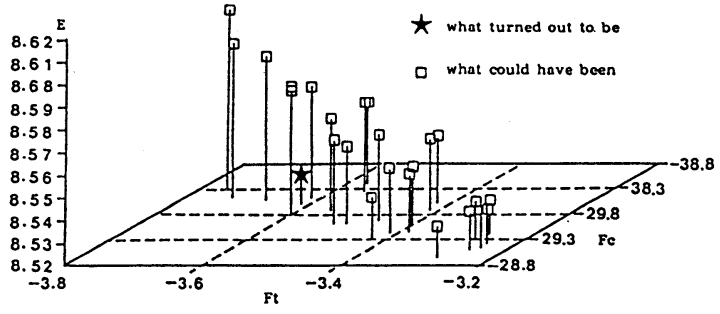

Fig. 4 A Three Dimentional Trade-off Pareto Diagram Source: Nakamura, M., et al... An Internctive Sewerage Planning Mlodel for Examining Interim Investment Strategies vs. Wasteiond
Reduction Profiles. Environmental Systems Research Vol. 19.

application to the planning situation defined for a particular point in time in the past leading to the present, and if the present relative position in the objective space were known (what turned out to be), then the relative position of the present situation represent "regrets", that the choice would have been better otherwise (as compared to what could have been).

The problems to overcome, then seem such items as (a), (b), (c) above, but item (a) and (b) are not of any greater magnitude than those shown under section 3.2, A, B, and C. As the number of objectives increases, item (c) would become quite limiting. But for most problems that we face, the number of objectives greater than three would be quite counter-intuitive anyway. The potential of retrospective application of multiobjective analysis, therefore, seems worth exploring further.

The prescriptive retrospecctive analysis and the descriptive retrospective analysis are complementary, and if used together, they are likely to enhance the understanding of the planning problem we face prospectively in future.

\section{References}

1. Chandler, C. G. (1981), Appropriate Technology for Planning Hydroelectric Power Projects in Nepal: The Need for Assumption Analysis, Center for Research in Water Resources, The University of Texas at Austin, Austin, Texas, U.S. A.

2. Bryson, J.M. (1990), Ring, P. Smith, A Transaction-Based Approach to Policy Intervention, Policy Science 23, pp205-229

3. Williamson, O.E. (1985), Ihe Economic Institutions of Capitalism, New York, The Free Press

4. Nickum, J.E. and Easter, K. W. (1989), Institutions and Water Use Conflicts: Concepts for Asian-Pacific Metropolises, UNCRD-EWC Joint Project on Water use Conflicts in Asian Metropolises, held at Lake Biwa Research Institute

5. Saliba, B.C. (1987), Do Water Markets "Work"?: Market Transfers and Trade-Offs in the Southwestern States, Water Resources Research, Vol.23, No.7, pp1113-1122

6. Linstone, H. A. (1984), Multiple Perspectives for Decision Making, New York, Elsevier Science Publishing Co., Inc. 